\title{
Network Topology Independent Multi-Agent Dynamic Optimal Power Flow for Microgrids with Distributed Energy Storage Systems
}

Morstyn, Thomas; Hredzak, Branislav; Agelidis, Vassilios G.

Published in:

IEEE Transactions on Smart Grid

Link to article, DOI:

10.1109/TSG.2016.2631600

Publication date:

2018

Document Version

Peer reviewed version

Link back to DTU Orbit

Citation (APA):

Morstyn, T., Hredzak, B., \& Agelidis, V. G. (2018). Network Topology Independent Multi-Agent Dynamic Optimal Power Flow for Microgrids with Distributed Energy Storage Systems. IEEE Transactions on Smart Grid, 9(4), 3419-3429. https://doi.org/10.1109/TSG.2016.2631600

\section{General rights}

Copyright and moral rights for the publications made accessible in the public portal are retained by the authors and/or other copyright owners and it is a condition of accessing publications that users recognise and abide by the legal requirements associated with these rights.

- Users may download and print one copy of any publication from the public portal for the purpose of private study or research.

- You may not further distribute the material or use it for any profit-making activity or commercial gain

- You may freely distribute the URL identifying the publication in the public portal 


\title{
Network Topology Independent Multi-Agent Dynamic Optimal Power Flow for Microgrids with Distributed Energy Storage Systems
}

\author{
Thomas Morstyn, Student Member, IEEE, Branislav Hredzak, Senior Member, IEEE, and \\ Vassilios G. Agelidis, Fellow, IEEE
}

\begin{abstract}
This paper proposes a multi-agent dynamic optimal power flow strategy for microgrids with distributed energy storage systems. The proposed control strategy uses a convex formulation of the AC dynamic optimal power flow problem developed from a d-q reference frame voltage-current model and linear power flow approximations. The convex dynamic optimal power flow problem is divided between autonomous agents and solved based on local information and neighbour-to-neighbour communication over a sparse communication network, using a distributed primal subgradient algorithm. Each agent is only required to solve convex quadratic sub-problems, for which robust and efficient solvers exist, making the control strategy suitable for receding horizon model predictive control. Also, the agent sub-problems require limited power network information and include only a subset of the centralised optimisation problem decision variables and constraints, providing scalability and data privacy. Unlike existing distributed optimal power flow methods, such as alternating direction method of multipliers, under the proposed control strategy the information required by each agent is independent of the communication network topology, providing increased flexibility and robustness. The performance of the proposed control strategy was verified for an AC microgrid with distributed lead-acid batteries and intermittent photovoltaic generation, using an RTDS Technologies real-time digital simulator.
\end{abstract}

Index Terms-Distributed optimisation, energy management system, energy storage systems, microgrid, model predictive control, multi-agent systems, dynamic optimal power flow, quadratic programming, renewable generation, tertiary control.

\section{INTRODUCTION}

$\mathbf{T}$ ECHNOLOGICAL developments and increased scales of production have dramatically reduced the costs of distributed renewable generation sources and energy storage (ES) systems. If properly managed, these sources have the potential to increase network efficiency, energy reliability and security, and to reduce pollution [1].

The microgrid concept provides a framework for managing the integration of these distributed sources [2]. A microgrid is a collocated set of generation sources, loads and ES systems that are coordinated to achieve autonomous operation. Since the microgrid can operate autonomously, it can be controlled

T. Morstyn and B. Hredzak are with the the School of Electrical Engineering and Telecommunications at The University of New South Wales (UNSW Australia), Sydney, NSW 2052 Australia (email: t.morstyn@student.unsw.edu.au, b.hredzak@unsw.edu.au).

V. G. Agelidis is with the Department of Electrical Engineering at the Technical University of Denmark (DTU), 4000 Roskilde, Denmark (email: vasagel@elektro.dtu.dk). as a dispatchable source when connected to the main grid, and can continue to operate if islanded.

The standard hierarchical model for microgrid control includes a centralised energy management system, which solves the microgrid optimal power flow (OPF) problem and sends set-points to lower level decentralised controllers at each source [3]. The OPF problem considers how the distributed sources should be dispatched to achieve economic objectives, such as power loss minimisation, while satisfying constraints imposed by network power quality requirements and device operating limits [4].

The OPF problem is non-convex, making it computationally challenging. The computational complexity of the OPF problem is significantly increased when ES systems are considered, since their state of charge (SoC) must be optimised over a prediction horizon [5]. This is described as the dynamic OPF (DOPF) problem. If predictions of the renewable generation and load profiles are available, the DOPF problem can be used for real-time control with a receding horizon model predictive control (MPC) implementation [3].

The non-convex microgrid DOPF problem can be solved using dynamic programming [5], [6] or nonlinear programming [7]. However, the computational complexity of these strategies limits the number of ES systems and the solution time may be too long to respond to varying renewable generation sources. The DOPF problem can be simplified by considering a single ES system [8]-[12] or an ideal real power transfer model between ES systems [13], [14]. This provides a convex programming problem, for which fast robust solvers exist, but neglects the network topology. A DC power flow approximation can be used to provide a convex DOPF problem, assuming the network has high $\mathrm{X} / \mathrm{R}$ ratios [15]. In this case, the optimisation does not consider reactive power flows, line losses and bus voltage limits.

The communications and processing infrastructure required for a centralised control strategy may be impractical for future microgrids made up of many small distributed renewable sources and ES systems [16]. Also, data centralisation introduces security and privacy concerns [17].

This motivates the use of multi-agent control. Under a multi-agent control strategy, autonomous agents use local information and neighbour-to-neighbour communication over a sparse communication network to achieve cooperative objectives [18]. Multi-agent control strategies can provide increased scalability, security and robustness over centralised 
control strategies [19]. Multi-agent control has been used in microgrids for secondary control [20]-[22] and SoC balancing between distributed ES systems [23]-[27].

The main advantages of a multi-agent DOPF strategy, over a centralised one, can be summarised as: (i) a single point of failure is removed, (ii) processing and data storage requirements can be divided between smaller, low cost distributed processors, (iii) distributed sources can join/leave the network without having to update a central coordinator, (iv) distributed sources can cooperate, while preserving data privacy and (v) the network cost can be reduced due to shorter link distances needed for only neighbour-to-neighbour communication.

Multi-agent DOPF strategies are presented in [28]-[31]. These are based on different simplifications of the DOPF problem, and different communication architectures. In [28], a convex DOPF problem is solved through iterative optimisation sub-problems distributed between microgrid agents, but a central controller is still required. Multi-agent DOPF strategies have also been presented based on dual decomposition algorithms [29] and alternating direction method of multipliers (ADMM) [30]. These strategies provide scalability since processing is divided between sparsely connected agents. However, the robustness and flexibility of a fully distributed multi-agent strategy are not provided, since each agent's role and the communication network between them must match the underlying power network topology. Also, [29] relies on the $\mathrm{X} / \mathrm{R}$ ratios of all lines being closely matched, and [30] uses the DC power flow approximation, which requires high $\mathrm{X} / \mathrm{R}$ ratios. These assumptions do not necessarily hold for low voltage microgrids. For example, the $\mathrm{X} / \mathrm{R}$ ratios for the benchmark $\mathrm{LV}$ microgrid from [32] vary from 0.0255 to 0.7028 . The strategy presented in [31] is fully distributed, but is based on an ideal real power transfer model which does not consider the power network topology.

This paper proposes a multi-agent DOPF strategy for microgrids with distributed ES systems and renewable generation sources. Under the proposed control strategy, autonomous agents use local information and neighbour-to-neighbour communication over a sparse network to reach agreement on the optimal power flows that minimise power consumption, considering the capacity of the distributed ES systems and renewable sources. The proposed control strategy is based on a convex formulation of the AC microgrid DOPF problem, obtained from a $\mathrm{d}-\mathrm{q}$ reference frame voltage-current model and linear power flow approximations. The convex formulation does not assume real and reactive power flows are decoupled, so line losses and voltage constraints can be explicitly considered. The convex formulation allows the DOPF problem to be divided between autonomous microgrid agents and solved using a distributed primal subgradient algorithm. Compared to the centralised optimisation problem, the agent sub-problems contain only a subset of the decision variables and constraints, and require only local ES system SoC estimates, local renewable generation predictions and limited power network information. This provides scalability, and a basis for data privacy and security between the agents (e.g. households with local PV and ES systems). Unlike existing distributed OPF methods, such as ADMM, the power network information required by each agent is independent of the communication network between them, and the agents will reach agreement as long as they are periodically connected. This has advantages in terms of flexibility and robustness to communication failures. The proposed control strategy uses a receding horizon MPC implementation, making it suitable for real-time control of a microgrid with intermittent renewable generation sources. The proposed multi-agent DOPF strategy has been integrated with low level decentralised droop control to share any power imbalance resulting from small mismatches between the agents' decision variables. The performance of the proposed control strategy was verified for a microgrid with intermittent PV generation and lead-acid battery ES systems, using an RTDS Technologies real-time digital simulator.

The rest of this paper is organised as follows. Section II describes the principle of operation of the proposed control strategy. The convex DOPF formulation is presented in Section III. The proposed multi-agent based control strategy is developed in Section IV by dividing the DOPF problem between the microgrid agents. Real-time simulation results demonstrating the performance of the proposed control strategy are presented in Section V. Section VI concludes the paper.

\section{PRINCIPLE OF OPERATION}

This study considers an islanded microgrid with distributed PV and battery ES systems, interfaced with the microgrid through voltage source converters (VSC). To achieve autonomous operation, the microgrid power balance must be maintained at all times. The distributed microgrid sources operate under the standard decentralised $f-P, V-Q$ droop control, which provides load sharing between the sources without requiring time-critical communication links [33].

$$
\begin{aligned}
\omega_{i} & =\omega_{0}-m_{P i}\left(P_{v s c i}-P_{v s c i}^{*}\right), \\
V_{o i} & =V_{o i}^{*}-n_{Q i}\left(Q_{v s c i}-Q_{v s c i}^{*}\right) .
\end{aligned}
$$

$\omega_{0}$ is the nominal microgrid frequency, $P_{v s c i}$ and $Q_{v s c i}$ are the real and reactive powers, $m_{P i}$ and $n_{Q i}$ are the droop coefficients, $P_{v s c i}^{*}$ and $Q_{v s c i}^{*}$ are the VSC real and reactive power references and $V_{o i}^{*}$ is the nominal output voltage magnitude. The generated frequency reference $\omega_{i}$ and voltage reference $V_{o i}$ are sent to lower level VSC voltage and current controllers.

The proposed multi-agent DOPF strategy is introduced at a higher level, to optimise the energy flows between the battery ES systems. The microgrid has autonomous agents, which collectively have information on the microgrid power network topology, renewable generation predictions and battery SoC estimates, but each agent only has access to a subset of this information.

The multi-agent DOPF strategy is implemented using receding horizon MPC, making it suitable for real-time control. A standard selection for the DOPF sampling period in microgrids with PV generation is 5 minutes [7]. Each sampling instant, the agents obtain updated local renewable generation predictions and SoC estimates. Between sampling instants, the agents cooperatively solve the DOPF problem based on the distributed primal subgradient algorithm from [34]. At each communication sub-interval, the agents solve reduced 


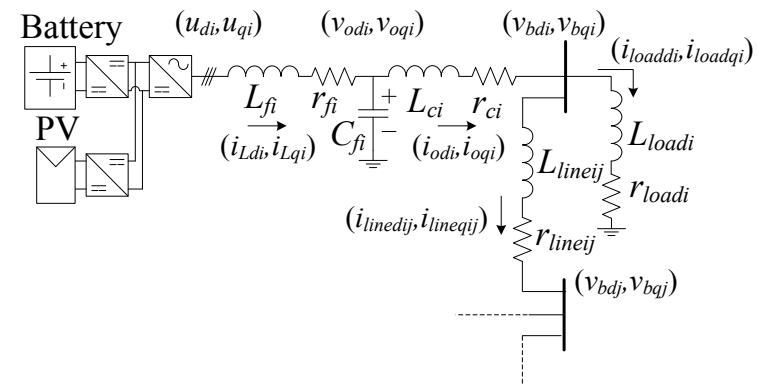

Fig. 1. Two bus microgrid segment with a PV and battery ES system, an RL load and an RL line. DC-DC converters interface the PV source and battery with the DC link of the VSC. The VSC is connected to the microgrid through an LCL filter.

size optimisation sub-problems (with a reduced number of decision variables and constraints compared with the centralised problem) using local information and information received from their neighbours in a sparse communication network. The agents converge towards the optimal solution as long as the communication network between them provides periodic connectivity, giving a measure of robustness to communication failures.

At the end of each MPC sampling interval, the agents collocated with distributed PV and battery ES systems use their local decision variables to generate a real power reference, reactive power reference and nominal output voltage for their local VSCs. These are supplied to the lower level droop control. Then, the MPC prediction horizon recedes by a step, the agents obtain updated renewable generation predictions and battery SoC estimates, and begin solving the next sampling interval's optimisation.

It is desirable to implement the proposed multi-agent DOPF strategy with as few communication sub-intervals as possible to reduce the computation time and amount of exchanged communication data. However, with a limited number of subintervals, the agents may not converge to an exact solution, and small mismatches between the agents' decision variables may result in a power imbalance. Microgrid disturbances (such as converter failures) may also result in a power imbalance. In the case of a power imbalance, the lower level decentralised droop control will share the microgrid load between the remaining VSCs.

\section{Convex Dynamic Optimal Power Flow}

In this section, the DOPF problem is formulated based on a static synchronous d-q reference frame voltage-current model. Power flow linearisations are introduced to obtain a convex optimisation problem.

\section{A. Microgrid Modelling}

Consider an islanded microgrid with distributed PV and battery ES systems, RL loads and RL lines. Let $S_{b u s}=$ $\left\{1, \ldots, N_{\text {bus }}\right\}$ be the set of microgrid buses and $S_{\text {batt }} \subseteq S_{\text {bus }}$ be the subset of buses with PV and battery ES systems. Let $S_{\text {line }}$ be the set of line current flow directions, where $(i, j) \in S_{\text {line }}$ and $(j, i) \in S_{\text {line }}$ if there is a line between bus $i$ and bus $j$. Let $\mathcal{N}_{i}^{P}$ be the power network neighbours of bus $i$, where $j \in \mathcal{N}_{i}^{P}$ if $(i, j) \in S_{\text {line }}$. Fig. 1 shows a two bus segment of the microgrid. The microgrid state equations are given by [33],

$$
\begin{aligned}
& L_{f i}\left[\begin{array}{c}
i_{L d i} \\
i_{L q i}
\end{array}\right]=-A_{i_{L i}}\left[\begin{array}{l}
i_{L d i} \\
i_{L q i}
\end{array}\right]+\left[\begin{array}{l}
u_{d i} \\
u_{q i}
\end{array}\right]-\left[\begin{array}{l}
v_{o d i} \\
v_{o q i}
\end{array}\right], \\
& C_{f i}\left[\begin{array}{l}
v_{o d i} \\
v_{o q i}
\end{array}\right]=-A_{v_{o i}}\left[\begin{array}{l}
v_{o d i} \\
v_{o q i}
\end{array}\right]+\left[\begin{array}{c}
i_{L d i} \\
i_{L q i}
\end{array}\right]-\left[\begin{array}{c}
i_{o d i} \\
i_{o q i}
\end{array}\right], \\
& L_{c i}\left[\begin{array}{c}
i_{o d i} \\
i_{\text {oqi }}
\end{array}\right]=-A_{i_{\text {oi }}}\left[\begin{array}{c}
i_{\text {odi }} \\
i_{\text {oqi }}
\end{array}\right]+\left[\begin{array}{c}
v_{\text {odi }} \\
v_{\text {oqi }}
\end{array}\right]-\left[\begin{array}{c}
v_{b d i} \\
v_{b q i}
\end{array}\right], i \in S_{\text {batt }}, \\
& L_{\text {loadi }}\left[\begin{array}{c}
i_{\text {loaddi }} \\
i_{\text {loadqi }}
\end{array}\right]=-A_{i_{\text {loadi }}}\left[\begin{array}{c}
i_{\text {loaddi }} \\
i_{\text {loadqi }}
\end{array}\right]+\left[\begin{array}{l}
v_{\text {bdi }} \\
v_{\text {bqi }}
\end{array}\right], i \in S_{\text {bus }}, \\
& L_{\text {lineij }}\left[\begin{array}{c}
i_{\text {linedij }} \\
i_{\text {lineqij }}
\end{array}\right]=-A_{i_{\text {lineij }}}\left[\begin{array}{l}
i_{\text {linedij }} \\
i_{\text {lineqij }}
\end{array}\right]+\left[\begin{array}{l}
v_{b d i} \\
v_{b q i}
\end{array}\right]-\left[\begin{array}{l}
v_{b d j} \\
v_{b q j}
\end{array}\right], \\
& (i, j) \in S_{\text {line }} \\
& A_{i_{L i}}=\left[\begin{array}{cc}
r_{f i} & -\omega_{0} L_{f i} \\
\omega_{0} L_{f i} & r_{f i}
\end{array}\right], A_{v_{o i}}=\left[\begin{array}{cc}
0 & -\omega_{0} C_{f i} \\
\omega_{0} C_{f i} & 0
\end{array}\right], \\
& A_{i_{o i}}=\left[\begin{array}{cc}
r_{c i} & -\omega_{0} L_{c i} \\
\omega_{0} L_{c i} & r_{c i}
\end{array}\right], A_{i_{\text {loadi }}}=\left[\begin{array}{cc}
r_{\text {loadi }} & -\omega_{0} L_{\text {loadi }} \\
\omega_{0} L_{\text {loadi }} & r_{\text {loadi }}
\end{array}\right], \\
& A_{i_{\text {lineij }}}=\left[\begin{array}{cc}
r_{\text {lineij }} & -\omega_{0} L_{\text {lineij }} \\
\omega_{0} L_{\text {lineij }} & r_{\text {lineij }}
\end{array}\right] \text {. }
\end{aligned}
$$

Respectively, $\left(i_{L d i}, i_{L q i}\right), \quad\left(i_{o d i}, i_{o q i}\right), \quad\left(u_{d i}, u_{q i}\right) \quad$ and $\left(v_{\text {odi }}, v_{\text {oqi }}\right)$ are the d-q components of the inductor current, output current, filter input voltage and output voltage of the PV and battery ES system VSC at bus $i$. The d-q components of the voltage and load current for bus $i$ are $\left(v_{b d i}, v_{b q i}\right)$ and $\left(i_{\text {loaddi }}, i_{\text {loadq } i}\right)$. The $\mathrm{d}-\mathrm{q}$ components for the line current from bus $i$ to bus $j$ are $\left(i_{\text {linedij }}, i_{\text {lineqij }}\right)$.

The proposed DOPF strategy controls the microgrid by supplying real power, reactive power and RMS voltage references to the VSCs each sampling instant, based on SoC estimates and predictions of the PV generation. Since the DOPF strategy operates on a much slower time-scale than the microgrid voltage-current dynamics, a static voltage-current model can be used to formulate the optimisation problem.

$$
\begin{aligned}
& {\left[\begin{array}{l}
i_{\text {loaddi }} \\
i_{\text {loadqi }}
\end{array}\right]=A_{i_{\text {loadi }}}^{-1}\left[\begin{array}{l}
v_{\text {bdi }} \\
v_{\text {bqi }}
\end{array}\right], i \in S_{\text {bus }},} \\
& {\left[\begin{array}{l}
i_{\text {linedij }} \\
i_{\text {lineqij }}
\end{array}\right]=A_{i_{\text {lineij }}}^{-1}\left(\left[\begin{array}{l}
v_{\text {bdi }} \\
v_{\text {bqi }}
\end{array}\right]-\left[\begin{array}{l}
v_{\text {bdj }} \\
v_{\text {bqj }}
\end{array}\right]\right),(i, j) \in S_{\text {line }},} \\
& {\left[\begin{array}{l}
i_{\text {odi }} \\
i_{\text {oqi }}
\end{array}\right]=\left[\begin{array}{l}
i_{\text {loaddi }} \\
i_{\text {loadqi }}
\end{array}\right]+\sum_{j \in \mathcal{N}_{i}^{P}}\left[\begin{array}{l}
i_{\text {linedij }} \\
i_{\text {lineqij }}
\end{array}\right],} \\
& {\left[\begin{array}{l}
v_{\text {odi }} \\
v_{\text {oqi }}
\end{array}\right]=\left[\begin{array}{l}
v_{\text {bdi }} \\
v_{\text {bqi }}
\end{array}\right]+A_{i_{\text {oi }}}\left[\begin{array}{l}
i_{\text {odi }} \\
i_{\text {oqi }}
\end{array}\right]} \\
& {\left[\begin{array}{l}
i_{\text {Ldi }} \\
i_{\text {Lqi }}
\end{array}\right]=A_{v_{\text {oi }}}\left[\begin{array}{l}
v_{\text {odi }} \\
v_{\text {oqi }}
\end{array}\right]+\left[\begin{array}{l}
i_{\text {odi }} \\
i_{\text {oqi }}
\end{array}\right],} \\
& {\left[\begin{array}{l}
u_{\text {di }} \\
u_{\text {qi }}
\end{array}\right]=\left[\begin{array}{l}
v_{\text {odi }} \\
v_{\text {oqi }}
\end{array}\right]+A_{i_{L i}}\left[\begin{array}{l}
i_{\text {Ldi }} \\
i_{\text {Lqi }}
\end{array}\right], i \in S_{\text {batt }} .}
\end{aligned}
$$

Let $\mathbf{v}_{b d q}=\left[\begin{array}{lllll}v_{b d 1} & v_{b q 1} & \cdots & v_{b d N_{b u s}} & v_{b q N_{b u s}}\end{array}\right]^{T}$ be the vector of microgrid bus voltages. From the static d-q voltage-current model (3), the microgrid voltages and currents can be ex- 


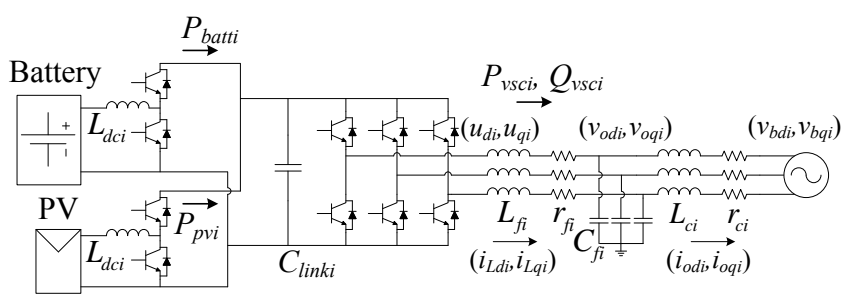

Fig. 2. PV and battery ES system architecture. The PV source and battery are interfaced through DC-DC converters to the VSC DC link. The VSC is connected to the microgrid through an LCL filter.

pressed as linear functions of the bus voltages.

$$
\begin{aligned}
& {\left[i_{\text {loaddi }} i_{\text {loadqi }}\right]^{T}=G_{i_{\text {loadi }}} \mathbf{v}_{b d q}, i \in S_{\text {bus }},} \\
& {\left[\begin{array}{ll}
i_{\text {linedij }} & i_{\text {lineqij }}
\end{array}\right]^{T}=G_{i_{\text {lineij }}} \mathbf{v}_{b d q},(i, j) \in S_{\text {line }},} \\
& {\left[\begin{array}{ll}
i_{L d i} & i_{L q i}
\end{array}\right]^{T}=G_{i_{L i}} \mathbf{v}_{b d q},\left[\begin{array}{ll}
u_{d i} & u_{q i}
\end{array}\right]^{T}=G_{u_{i}} \mathbf{v}_{b d q},} \\
& {\left[\begin{array}{ll}
i_{\text {odi }} & i_{\text {oqi }}
\end{array}\right]^{T}=G_{i_{o i}} \mathbf{v}_{b d q}, \quad\left[\begin{array}{ll}
v_{\text {odi }} & v_{o q i}
\end{array}\right]^{T}=G_{v_{o i}} \mathbf{v}_{b d q}, \quad i \in S_{\text {batt }} .}
\end{aligned}
$$

Fig. 2 shows a typical architecture for a PV and battery ES system. The PV source and battery are interfaced through DC-DC converters to the DC link of a three phase VSC. The VSC is connected to the microgrid through an LCL filter. The $\mathrm{PV}$ generation source is controlled to operate at its maximum power point, while the VSC is controlled using decoupled d-q voltage and current controllers to achieve the frequency and voltage references set by the droop control. The battery is operated to maintain the VSC DC link voltage, and therefore supplies the extra power required to balance the PV generation with the power exported by the VSC.

The following model is widely used for the battery SoC dynamics [10], [11], [28]-[31].

$$
\begin{aligned}
S o C_{i}(k+1) & =S o C_{i}(k)-\frac{T_{s}}{E_{b a t t i}} P_{b a t t i}(k), \\
P_{b a t t i}(k) & =P_{v s c i}(k)-P_{p v i}(k), \quad i \in S_{b a t t} .
\end{aligned}
$$

$E_{\text {batti }}$ is the battery energy capacity, $T_{s}$ is the sampling period, $P_{\text {batti }}(k)$ is the battery output power and $P_{p v i}(k)$ is the PV source output power. Note that this model does not include the limited charging and discharging efficiency of the battery.

The VSC real output power is given by,

$$
P_{v s c i}(k)=i_{L d i}(k) u_{d i}(k)+i_{L q i}(k) u_{q i}(k) .
$$

The following approximation for the VSC real output power is used to obtain a linear SoC model, based on a nominal operating point of $\left(I_{L d i}, I_{L q i}\right)$ for the VSC inductor current and $\left(U_{d i}, U_{q i}\right)$ for the VSC filter input voltage.

$$
\begin{gathered}
\tilde{P}_{v s c i}(k)=\left[\begin{array}{cc}
I_{L d i} & \left.I_{L q i}\right]
\end{array}\right] G_{u_{i}} \mathbf{v}_{b d q}(k)+\left[U_{d i} U_{q i}\right] G_{i_{L i}} \mathbf{v}_{b d q}(k) \\
-I_{L d i} U_{d i}-I_{L q i} U_{q i}
\end{gathered}
$$

Constant power loads can be included in the d-q reference frame voltage-current model using similar power flow approximations. This is described in the Appendix.

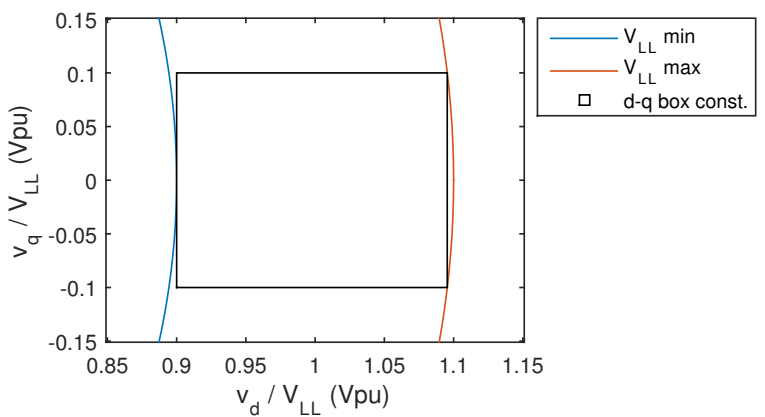

Fig. 3. Line to line RMS bus voltage constraints and conservative box constraints.

\section{B. Convex Dynamic Optimal Power Flow Formulation}

Let $\tau=\left\{0, \ldots, N_{p}-1\right\}$ be the optimisation prediction horizon. The inputs of the optimisation are the battery SoC estimates $S o C_{i}(0), i \in S_{\text {batt }}$, and the predicted average PV generation during each sampling interval of the prediction horizon, $P_{\text {pvi }}(k), i \in S_{\text {batt }}, k \in \tau$.

In this study, the objective selected for the islanded microgrid is to minimise average power consumption, while operating the renewable generation sources at their maximum power point. Minimising power consumption can be desirable when energy supplies are limited. Conservation voltage reduction, i.e. controlling bus voltages towards the lower end of allowed limits to reduce power consumption, has been widely applied to achieve this [35]-[39]. Conservation voltage reduction is naturally provided by the DOPF strategy with a power consumption minimisation cost function.

The power consumed by the microgrid loads, VSC filters and lines, over the prediction horizon, are given by,

$$
\begin{aligned}
J_{\text {loadi }} & =\sum_{k \in \tau} r_{\text {loadi }}\left(i_{\text {loaddi }}^{2}(k)+i_{\text {loadqi }}^{2}(k)\right) \\
& =\sum_{k \in \tau} r_{\text {loadi }} \mathbf{v}_{b d q}^{T}(k) G_{i_{\text {loadi }}}^{T} G_{i_{\text {loadi }}} \mathbf{v}_{b d q}(k), \\
J_{\text {vsci }} & =\sum_{k \in \tau} r_{f i}\left(i_{\text {Ldi }}^{2}(k)+i_{L q i}^{2}(k)\right)+r_{c i}\left(i_{\text {odi }}^{2}(k)+i_{\text {oqi }}^{2}(k)\right) \\
& =\sum_{k \in \tau} \mathbf{v}_{b d q}^{T}(k)\left(r_{f i} G_{i_{L i}}^{T} G_{i_{L i}}+r_{c i} G_{i_{o i}}^{T} G_{i_{o i}}\right) \mathbf{v}_{b d q}(k), \\
J_{\text {lineij }} & =\sum_{k \in \tau} r_{\text {lineij }}\left(i_{\text {linedij }}^{2}(k)+i_{\text {lineqij }}^{2}(k)\right) \\
& =\sum_{k \in \tau} r_{\text {lineij }} \mathbf{v}_{b d q}^{T}(k) G_{i_{\text {lineij }}}^{T} G_{i_{\text {lineij }}} \mathbf{v}_{b d q}(k) .
\end{aligned}
$$

Alternative objectives can be considered depending on the microgrid. With constant power loads, power loss minimisation is a more suitable objective. In this case the $J_{\text {loadi }}$ terms would not be included in the optimisation cost function. In a microgrid with a mix of conventional generation sources and battery ES systems, it may be desirable to include battery depreciation in the cost function. This can be done using an Ah throughput model for battery lifetime degradation [40].

Let $V_{L L}$ be the nominal microgrid line to line voltage. Standard microgrid bus voltage limits are $0.9 \mathrm{pu}$ to $1.1 \mathrm{pu}$. This 
requires that,

$$
0.9 V_{L L} \leq \sqrt{v_{b d i}^{2}(k)+v_{b q i}^{2}(k)} \leq 1.1 V_{L L}, \quad i \in S_{b u s}, k \in \tau .
$$

Assuming nominal d-q bus voltages of $\left(V_{L L}, 0\right)$, the quadratic voltage constraints (11) can be approximated with the following box constraints,

$$
\begin{aligned}
& 0.9 V_{L L} \leq v_{b d i}(k) \leq 1.0954 V_{L L}, \\
& -0.1 V_{L L} \leq v_{b q i}(k) \leq 0.1 V_{L L}, i \in S_{b u s}, k \in \tau .
\end{aligned}
$$

Fig. 3 shows the limits on the RMS line to line bus voltages (11) in the $\mathrm{d}-\mathrm{q}$ reference frame and the approximate box constraints (12).

The VSCs should be kept within output power limits to ensure they are not overloaded. Approximate constraints are introduced on the VSC real output powers using (7).

$$
P_{v s c}^{\min } \leq \tilde{P}_{v s c i}(k) \leq P_{v s c}^{\max }, i \in S_{b a t t}, k \in \tau .
$$

Batteries suffer from significant lifetime deterioration when overcharged or undercharged [41]. Therefore, the batteries should be operated within SoC limits.

$$
\left[\begin{array}{c}
S o C_{i}^{\text {min }} \\
\vdots \\
S o C_{i}^{\text {min }}
\end{array}\right] \leq\left[\begin{array}{c}
S o C_{i}(1) \\
\vdots \\
S o C_{i}\left(N_{p}\right)
\end{array}\right] \leq\left[\begin{array}{c}
S o C_{i}^{\max } \\
\vdots \\
S o C_{i}^{\max }
\end{array}\right], i \in S_{\text {batt }}
$$

The battery SoC values over the prediction horizon can be expressed as affine functions of the initial $\mathrm{SoC}$, the microgrid bus voltages and the PV generation, using (5) and (7).

$$
\begin{aligned}
& {\left[\begin{array}{c}
S o C_{i}(1) \\
\vdots \\
S o C_{i}\left(N_{p}\right)
\end{array}\right]=\left[\begin{array}{c}
S o C_{i}(0) \\
\vdots \\
S o C_{i}(0)
\end{array}\right]+\left[\begin{array}{cccc}
1 & 0 & \cdots & 0 \\
\vdots & \ddots & \ddots & \vdots \\
\vdots & & \ddots & 0 \\
1 & \cdots & \cdots & 1
\end{array}\right]\left[\begin{array}{c}
g_{S o C i}(0) \\
\vdots \\
g_{S o C i}\left(N_{p}-1\right)
\end{array}\right]} \\
& +\left[\begin{array}{cccc}
B_{S o C i} & 0 & \cdots & 0 \\
\vdots & \ddots & \ddots & \vdots \\
\vdots & & \ddots & 0 \\
B_{S o C i} & \cdots & \cdots & B_{S o C i}
\end{array}\right]\left[\begin{array}{c}
\mathbf{v}_{b d q}(0) \\
\vdots \\
\mathbf{v}_{b d q}\left(N_{p}-1\right)
\end{array}\right] \\
& B_{S o C i}=\frac{-T_{s}}{E_{b a t t i}}\left(\left[\begin{array}{ll}
I_{L d i} & I_{L q i}
\end{array}\right] G_{u_{i}}+\left[\begin{array}{ll}
U_{d i} & U_{q i}
\end{array}\right] G_{i_{L i}}\right), \\
& g_{S o C i}(k)=\frac{T_{s}}{E_{b a t t i}}\left(I_{L d i} U_{d i}+I_{L q i} U_{q i}+P_{p v i}(k)\right) .
\end{aligned}
$$

The current flowing into each bus must be zero at each time interval to maintain the microgrid power balance. For buses with a battery ES system, any mismatch in the line and load currents will be reflected in the ES system output power. The following constraints must be introduced for buses without an ES system.

$$
\left(G_{i_{\text {loadi }}}+\sum_{j \in \mathcal{N}_{i}^{P}} G_{i_{\text {lineij }}}\right) \mathbf{v}_{b d q}(k)=0, i \in S_{\text {bus }}, i \notin S_{\text {batt }}, k \in \tau .
$$

Combining the power consumption functions (8), (9), (10) and constraints (12), (13), (14), (16), the centralised microgrid DOPF problem can be formulated as a convex quadratic program $(\mathrm{QP})$. Let the vector of decision variables be $x \in \mathbf{R}^{m}, m=2 N_{b u s} N_{p}$, the $\mathrm{d}$-q reference frame bus voltages over the optimisation prediction horizon, i.e. $x=\left[\begin{array}{lll}\mathbf{v}_{b d q}^{T}(0) & \cdots & \mathbf{v}_{b d q}^{T}\left(N_{p}-1\right)\end{array}\right]^{T}$. The DOPF problem is given by,

$$
\begin{array}{ll}
\underset{x \in \mathbf{R}^{m}}{\operatorname{minimise}} & \sum_{i \in S_{\text {bus }}} J_{\text {loadi }}+\sum_{i \in S_{\text {batt }}} J_{v s c i}+\sum_{(i, j) \in S_{\text {line }}} \frac{1}{2} J_{\text {lineij }} \\
\text { subject to } & (12),(13),(14),(16) .
\end{array}
$$

\section{Multi-Agent DOPF Strategy}

Consider autonomous microgrid agents $\mathcal{V}=\left\{1, \ldots, N_{\text {agt }}\right\}$ that cooperatively solve the DOPF using limited power network information and neighbour-to-neighbour communication.

The agents are connected by a sparse communication network, and share information at sub-intervals between the MPC sampling instants. The communication network is represented by a directed graph $\mathcal{G}(\mathcal{V}, \mathcal{E}(\kappa))$, with edges $\mathcal{E}(\kappa)$ during subinterval $\kappa$. An edge $(a, b) \in \mathcal{E}(\kappa)$ if there is a communication link allowing information to flow from agent $a$ to agent $b$. Let $\mathcal{N}_{a}(\kappa)$ be the neighbours of agent $a$, where $b \in \mathcal{N}_{a}(\kappa)$ if $(b, a) \in \mathcal{E}(\kappa)$. The graph's weighted adjacency matrix is given by $\mathcal{A}(\kappa)=\left[w_{a b}(\kappa)\right] \in \mathbf{R}^{N_{a g t} \times N_{a g t}}$. The adjacency matrix diagonals $w_{a a}(\kappa)=1-\sum_{b \in \mathcal{N}_{a}} w_{a b}(\kappa)$, and for $a \neq b$, link weight $w_{a b}(\kappa)>0$ if $(b, a) \in \mathcal{E}(\kappa)$ and $w_{a b}(\kappa)=0$ otherwise.

Under the following communication network conditions, the DOPF problem (17) can be divided between the autonomous agents and solved using the distributed primal subgradient algorithm from [34].

1) Non-Degeneracy: There exists $\beta>0$ such that $w_{a a}(\kappa)=1-\sum_{b \in \mathcal{N}_{a}} w_{a b}(\kappa) \geq \beta$, and $w_{a b}(\kappa) \in$ $\{0\} \cup[\beta, 1], a \neq b$, for all $\kappa \geq 0$.

2) Balanced Communication: $\sum_{a=1}^{N_{a g t}} w_{a b}(\kappa)=1$ and $\sum_{b=1}^{N_{a g t}} w_{a b}(\kappa)=1$, for all $\kappa \geq 0$.

3) Periodic Strong Connectivity: There is a positive integer $B$ such that the graph $\left(\mathcal{V}, \cup_{\kappa=0}^{B-1} \mathcal{E}\left(\kappa_{0}+\kappa\right)\right)$ is strongly connected for all $\kappa_{0} \geq 0$.

Each agent has access to a limited amount of power network information. Let each agent $a \in \mathcal{V}$ be assigned a subset of the PV and battery ES systems, $S_{\text {batt }}^{[a]} \subseteq S_{\text {batt }}$, buses $S_{b u s}^{[a]} \subseteq$ $S_{\text {bus }}$, and line current flow directions, $S_{\text {line }}^{[a]} \subseteq S_{\text {line }}$. The PV and battery ES systems are assigned such that, $\cup_{a \in \mathcal{V}} S_{b a t t}^{[a]}=$ $S_{\text {batt }}$. The buses assigned to agent $a$ include those with the assigned PV and battery ES systems, $S_{\text {bus }}^{[a]} \supseteq\left\{i \mid i \in S_{b a t t}^{[a]}\right\}$. All buses must be assigned to at least one agent, i.e. $\cup_{a \in \mathcal{V}} S_{b u s}^{[a]}=$ $S_{\text {bus }}$. The agents are assigned the line current flow directions that have one of their assigned buses as its origin, $S_{\text {line }}^{[a]} \supseteq$ $\left\{(i, j) \mid i \in S_{\text {bus }}^{[a]},(i, j) \in S_{\text {line }}\right\}$.

The information available to each agent depends on the PV and battery ES systems, buses and line current flow directions assigned to it. For each PV and battery ES $i \in S_{b a t t}^{[a]}$, the agent has access to the VSC filter impedances $A_{i_{L i}}, A_{v_{o i}}, A_{i_{o i}}$, the current battery SoC estimates $S_{o} C_{i}(0)$ and PV generation predictions $P_{p v i}(k), k \in \tau$. For each assigned bus $i \in S_{b u s}^{[a]}$, the agent has access to the load impedances $A_{i_{\text {loadi }}}$. For each assigned line current flow direction $(i, j) \in S_{\text {line }}^{[a]}$, the agent has access to the line impedances $A_{i_{\text {lineij }}}$. 
The DOPF cost function is divided between the agents based on their available power network information.

$$
J^{[a]}=\sum_{i \in S_{\text {bus }}^{[a]}} \frac{J_{\text {loadi }}}{N_{\text {busi }}^{a g t}}+\sum_{i \in S_{\text {batt }}^{[a]}} \frac{J_{\text {vsci }}}{N_{\text {batti }}^{a g t}}+\sum_{(i, j) \in S_{\text {line }}^{[a]}} \frac{1}{2} \frac{J_{\text {lineij }}}{N_{\text {lineij }}^{\text {agt }}} .
$$

$N_{\text {batti }}^{\text {agt }}, N_{\text {busi }}^{\text {agt }}, N_{\text {lineij }}^{\text {agt }}$ are the number of agents each PV and battery ES system, bus and line current flow direction are respectively assigned to.

Each agent is also assigned a subset of the DOPF constraints. The convex constraint set for agent $a$ is given by $X^{[a]}=\left\{x \in \mathbf{R}^{m} \mid(19),(20),(21),(22)\right\}$.

$$
\begin{aligned}
& 0.9 V_{L L} \leq v_{b d i}(k) \leq 1.0954 V_{L L}, \\
& -0.1 V_{L L} \leq v_{b q i}(k) \leq 0.1 V_{L L}, i \in S_{b u s}^{[a]} \cup_{j \in S_{b u s}^{[a]}} \mathcal{N}_{j}^{P}, k \in \tau, \\
& P_{v s c}^{\min } \leq \tilde{P}_{v s c i}(k) \leq P_{v s c}^{\max }, i \in S_{b a t t}^{[a]}, k \in \tau, \\
& A_{i_{\text {loadi }}}\left[\begin{array}{l}
v_{b d i}(k) \\
v_{b q i}(k)
\end{array}\right]+\sum_{j \in \mathcal{N}_{i}^{P}} A_{i_{\text {lineij }}}^{-1}\left(\left[\begin{array}{l}
v_{b d i}(k) \\
v_{b q i}(k)
\end{array}\right]-\left[\begin{array}{l}
v_{b d j}(k) \\
v_{b q j}(k)
\end{array}\right]\right)=0, \\
& i \in S_{\text {bus }}^{[a]}, \quad i \notin S_{\text {batt }}, k \in \tau, \\
& {\left[\begin{array}{c}
S o C_{i}^{\min } \\
\vdots \\
S o C_{i}^{\text {min }}
\end{array}\right] \leq\left[\begin{array}{c}
S o C_{i}(1) \\
\vdots \\
S o C_{i}\left(N_{p}\right)
\end{array}\right] \leq\left[\begin{array}{c}
S o C_{i}^{\max } \\
\vdots \\
S o C_{i}^{\max }
\end{array}\right], i \in S_{\text {batt }}^{[a]}}
\end{aligned}
$$

The sum of the individual agent cost functions are equal to the centralised DOPF problem cost function, and the intersection of the agent constraint sets include all of the DOPF problem constraints. Therefore, the central microgrid DOPF problem (17) is equivalent to the following separable convex QP,

$$
\begin{array}{ll}
\underset{x \in \mathbf{R}^{m}}{\operatorname{minimise}} & \sum_{a \in \mathcal{V}} J^{[a]} \\
\text { subject to } & x \in \cap_{a \in \mathcal{V}} X^{[a]} .
\end{array}
$$

Due to the sparse nature of power networks, only some of the microgrid bus voltages are relevant to each agent's cost function and constraint set. The number of decision variables for agent $a$ is given by $m^{[a]}=2\left|S_{b u s}^{[a]} \cup_{i \in S^{[a]}} \mathcal{N}_{i}^{P}\right| N_{p}$, the number of $\mathrm{d}$-q voltages over the prediction horizon for the buses assigned to the agent and those buses' neighbours.

A reduced size decision vector for agent $a$ can be obtained from a linear mapping $\mathcal{M}^{[a]}: \mathbf{R}^{m} \rightarrow \mathbf{R}^{m^{[a]}}, \tilde{x}^{[a]}=$ $\mathcal{M}^{[a]}\left(x^{[a]}\right)$. A reverse map is also defined for each agent, $\mathcal{M}^{\prime[a]}: \mathbf{R}^{m^{[a]}} \times \mathbf{R}^{m} \rightarrow \mathbf{R}^{m}, x^{[a]}=\mathcal{M}^{\prime[a]}\left(\tilde{x}^{[a]}, v^{[a]}\right)$ which maps the elements of a reduced decision vector $\tilde{x}^{[a]} \in \mathbf{R}^{m^{[a]}}$ back onto a full size decision vector $x^{[a]} \in \mathbf{R}^{m}$, and takes the remaining elements from $v^{[a]} \in \mathbf{R}^{m}$.

For each agent $a \in \mathcal{V}$, an appropriate cost function $\tilde{J}^{[a]}: \mathbf{R}^{m^{[a]}} \rightarrow \mathbf{R}$ is chosen such that $\tilde{J}^{[a]}\left(\mathcal{M}\left(x^{[a]}\right)\right)=$ $J^{[a]}\left(x^{[a]}\right), \forall x^{[a]} \in \mathbf{R}^{m}$ and constraint set $\tilde{X}^{[a]}=\left\{\tilde{x}^{[a]} \in\right.$ $\mathbf{R}^{m^{[a]}} \mid$ (19), (20), (21), (22)\}.

The distributed primal subgradient algorithm from [34] is used to solve the multi-agent DOPF problem. The algorithm has been modified to use the sparse structure of the power network so that each agent solves reduced size optimisation sub-problems.

Each agent maintains a local estimate of the full microgrid decision vector $x^{[a]}$ and initialises it so that $x^{[a]}(0) \in X^{[a]}$.

At each communication sub-interval, $\kappa \geq 0$, the agents share their decision vectors with their neighbours and take a convex combination,

$$
v^{[a]}(\kappa)=w_{a a}(\kappa) x^{[a]}(\kappa)+\sum_{b \in \mathcal{N}_{a}} w_{a b}(\kappa) x^{[b]}(\kappa) .
$$

The convex combination of decision vectors is reduced to the elements relevant to the agent sub-problem using the linear map,

$$
\tilde{v}^{[a]}(\kappa)=\mathcal{M}^{[a]}\left(v^{[a]}(\kappa)\right) .
$$

Each agent then updates their reduced size local decision vector by making a subgradient step from the convex combination to reduce their local cost function and projecting the result onto their local constraint set $\tilde{X}^{[a]}$,

$$
\tilde{x}^{[a]}(\kappa+1)=P_{\tilde{X}^{[a]}}\left[\tilde{v}^{[a]}(\kappa)-\alpha(\kappa) \nabla \tilde{J}^{[a]}\left(\tilde{v}^{[a]}(\kappa)\right)\right] .
$$

The updated reduced size decision vector and the convex combination of neighbour decision vectors are used to update the local full size microgrid decision vector, using the reverse map,

$$
x^{[a]}(\kappa+1)=\mathcal{M}^{\prime[a]}\left(\tilde{x}^{[a]}(\kappa+1), v^{[a]}(\kappa)\right) .
$$

$\alpha(\kappa)$ is the distributed primal subgradient algorithm step size. $\alpha(\kappa)$ should be chosen so that [34],

$$
\lim _{\kappa \rightarrow \infty} \alpha(\kappa)=0, \sum_{\kappa=0}^{\infty} \alpha(\kappa)=\infty, \sum_{\kappa=0}^{\infty} \alpha^{2}(\kappa)<\infty .
$$

Since each agent's local cost function $\tilde{J}^{[a]}$ and constraint set $\tilde{X}^{[a]}$ are independent of decision variables in $x^{[a]}$ that are not in $\tilde{x}^{[a]}$, the proof of convergence from [34] is valid for the modified algorithm. Therefore, the local agent decision vectors approach the optimal solution $x^{*}$ of the centralised DOPF problem (17), $\lim _{\kappa \rightarrow \infty}\left\|x^{[a]}(\kappa)-x^{*}\right\|_{2}=0, \forall a \in \mathcal{V}$.

Each agent solves the projection problem (26) using the following convex QP,

$$
\begin{aligned}
& \underset{\tilde{w}^{a]}(\kappa+1) \in \mathbf{R}^{m}[a]}{\operatorname{minimize}}\left\|\tilde{x}^{[a]}(\kappa+1)-\tilde{v}^{[a]}(\kappa)+\alpha(\kappa) \nabla \tilde{J}^{[a]}\left(\tilde{v}^{[a]}(\kappa)\right)\right\|_{2}^{2} \\
& \text { subject to } \quad \tilde{x}^{[a]}(\kappa+1) \in \tilde{X}^{[a]} .
\end{aligned}
$$

The proposed multi-agent DOPF strategy is implemented using receding horizon MPC. At each optimisation sampling instant, the microgrid agents obtain estimates of their local battery SoC and predictions of the average renewable generation for each interval of the prediction horizon. Under the distributed primal subgradient algorithm, the agents iteratively solve their local projection problems and combine their local decision vector estimates so that they cooperatively converge towards a solution to the microgrid DOPF problem. After the number of communication sub-intervals allowed by the duration of the MPC sampling period, the agents collocated with PV and battery ES systems generate a local VSC real power reference, reactive power reference and nominal output 
TABLE I

REAL-TIME SimUlation PARAMETERS

\begin{tabular}{llllll}
\hline$N_{p}$ & 3 & $T_{s}$ & $5 \mathrm{~min}$ & $\omega_{0}$ & $50 \mathrm{~Hz}$ \\
$V_{L L}$ & $415 \mathrm{~V}$ & $V^{\max }$ & $1.1 \mathrm{~V}_{L L}$ & $V^{\text {min }}$ & $0.9 \mathrm{~V}_{L L}$ \\
$E_{b a t t}$ & $20 \mathrm{kWh}$ & $S_{o C^{\max }}$ & $100 \%$ & $S_{0} C^{\text {min }}$ & $40 \%$ \\
$P_{\text {vax }}^{\text {max }}$ & $6 \mathrm{~kW}$ & $P_{\text {min }}^{\text {min }}$ & $-6 \mathrm{~kW}$ & $m_{P}$ & $2.1 \times 10^{-5}$ \\
$n_{Q}$ & $2.8 \times 10^{-4}$ & $f_{v s c}$ & $2 \mathrm{kHz}$ & $f_{d c d c}$ & $1 \mathrm{kHz}$ \\
$r_{f}$ & $0.01 \Omega$ & $L_{f}$ & $5 \mathrm{mH}$ & $C_{f}$ & $68 \mu \mathrm{F}$ \\
$r_{c}$ & $0.01 \Omega$ & $L_{c}$ & $5 \mathrm{mH}$ & $C_{l i n k}$ & $3.4 \mathrm{mF}$ \\
$L_{d c}$ & $150 \mathrm{mH}$ & & & & \\
\hline
\end{tabular}

TABLE II

Central and Multi-Agent Dopf Problem Sizes

\begin{tabular}{lcc}
\hline Number of: & $\begin{array}{c}\text { Multi-Agent } \\
\text { DOPF Agents }\end{array}$ & $\begin{array}{c}\text { Central } \\
\text { DOPF }\end{array}$ \\
\hline Buses & 1 & 9 \\
PV+Batt. Systems & $\leq 1$ & 5 \\
Line Directions & $\leq 3$ & 16 \\
Decision Variables & $\leq 24$ & 54 \\
Constraints & $\leq 54$ & 192 \\
\hline
\end{tabular}

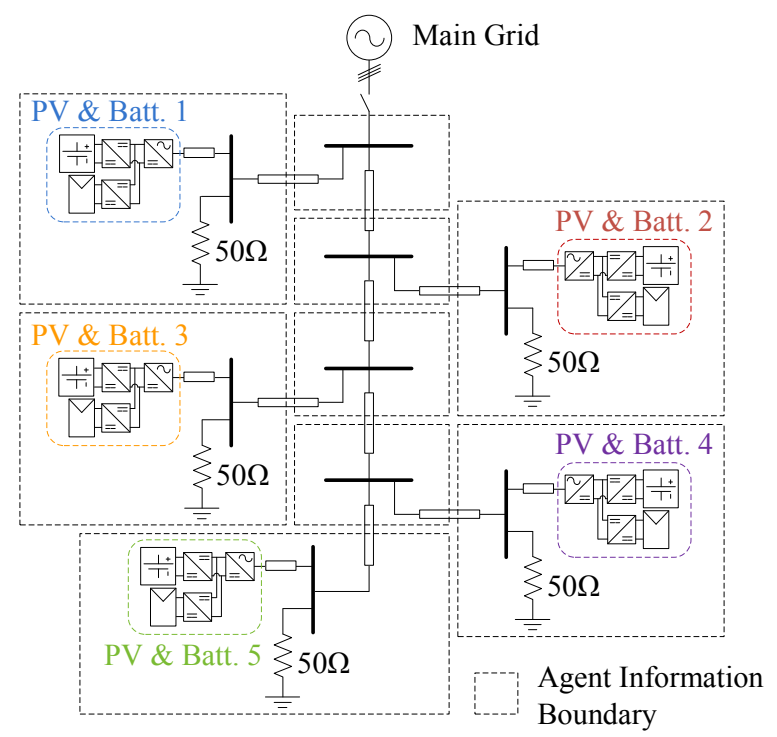

voltage magnitude for the first interval of the prediction horizon by solving,

$$
\begin{aligned}
P_{v s c i}^{*}(0) & =u_{d i}^{[a]}(0) i_{L d i}^{[a]}(0)+u_{q i}^{[a]}(0) i_{L q i}^{[a]}(0), \\
Q_{v s c i}^{*}(0) & =u_{d i}^{[a]}(0) i_{L q i}^{[a]}(0)-u_{q i}^{[a]}(0) i_{L d i}^{[a]}(0), \\
V_{o i}^{*}(0) & =\sqrt{v_{o d i}^{[a]}(0)^{2}+v_{o q i}^{[a]}(0)^{2}} .
\end{aligned}
$$

The agents send the references to the lower level droop controllers of their PV and battery ES system VSCs. At the next optimisation sampling instant, the prediction horizon is incremented by a step, and new references are obtained using the updated $\mathrm{SoC}$ estimates and renewable generation predictions.

\section{RESULTS}

To verify the performance of the proposed multi-agent DOPF strategy, real-time simulations were carried out for the European residential benchmark low voltage microgrid from [32]. As shown in Fig. 4, the microgrid is islanded from the main grid, and has five PV and battery ES systems and nine buses. The microgrid has five $50 \Omega$ loads $(5 \times 3.44 \mathrm{~kW}$ nominal load).

Two case studies were carried out: (a) the microgrid operating under the centralised DOPF strategy, and (b) the microgrid operating under the multi-agent DOPF strategy. For the multiagent case study, there are nine agents, each associated with one of the microgrid buses. The agent information boundaries are shown in Fig. 4. The microgrid agents are fully connected by a communication network, i.e. there are links allowing neighbour-to-neighbour communication between all of the agents. To demonstrate the robustness of the proposed multi-agent control strategy to communication failures, each communication link is given a $5 \%$ chance of failure at each communication sub-interval, representing packet losses.

The simulations were completed using an RTDS technologies real-time digital simulator, with switching converter

Fig. 4. Islanded benchmark low voltage microgrid with five PV and battery ES systems and nine agents. The agent boundaries show the limits of the power network information available to them.

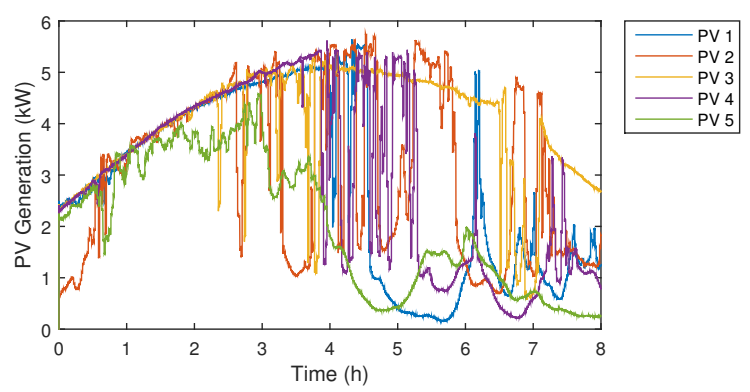

Fig. 5. PV generation source output powers for the centralised and multiagent DOPF case studies.

models and nonlinear battery models from [42]. The use of switching converter models and battery models capturing the fast and slow time-scale battery voltage and SoC dynamics allows the proper interaction between the different microgrid control levels to be verified.

The real-time simulation parameters are provided in Table I. Each PV and battery ES system has a $5 \mathrm{~kW}$ PV generation source and a $20 \mathrm{kWh} 416 \mathrm{~V}$ lead-acid battery. The PV generation source maximum power point was calculated based on irradiance and temperature data with one minute resolution from the NREL Baseline Measurement Station in Colorado. Data was taken from $8 \mathrm{am}$ to $4 \mathrm{pm}$ for July 1 st to July 5 th, 2015, and each day's data was used for one of the five PV sources.

The centralised and multi-agent DOPF MPC strategies were formulated with 5 minute sampling periods (like the centralised DOPF strategy in [7]), so they could respond to changes in PV generation. A prediction horizon of 15 minutes (3 sampling intervals) was used, based on the expected 


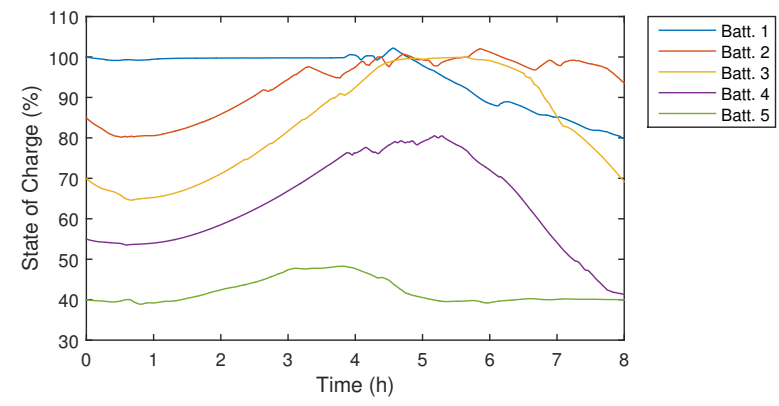

(a) Centralised DOPF.

Fig. 6. Battery SoC levels.

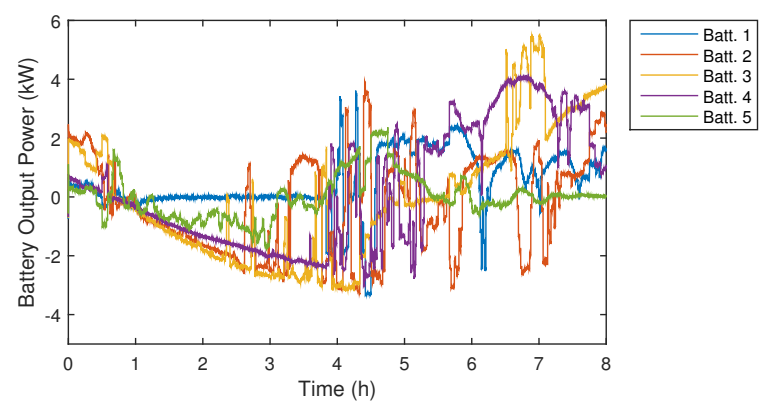

(a) Centralised DOPF.

Fig. 7. Battery output powers.

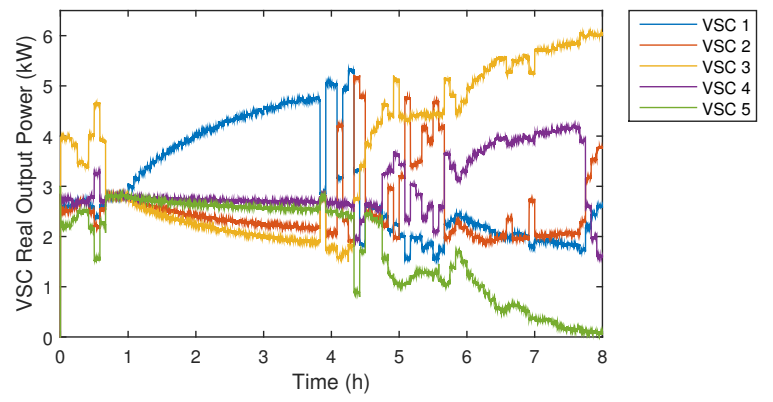

(a) Centralised DOPF.

Fig. 8. VSC real output powers.

availability of PV predictions from sky imaging sensors [43]. It is assumed that agents that are assigned PV and battery ES systems have access to accurate estimates the battery SoC and the average PV generation for each interval of the prediction horizon.

Table II compares the size of the central DOPF problem to the sizes of the agent sub-problems. The centralised DOPF problem has 54 decision variables and 192 constraints (treating each affine equality constraint as two inequalities). For the multi-agent DOPF strategy, the agent sub-problems have at most 24 decision variables and 54 constraints. The reduced number of decision variables and constraints limits the processing infrastructure and information required by each agent, improving scalability.

IBM's CPLEX solver in MATLAB was used to solve the QP problems for the centralised DOPF strategy and the

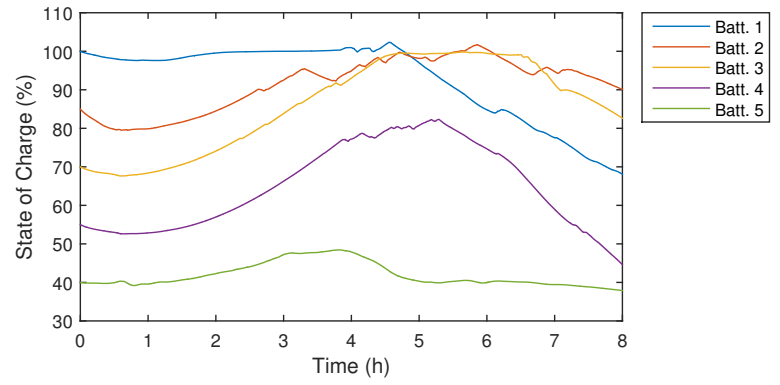

(b) Multi-Agent DOPF.

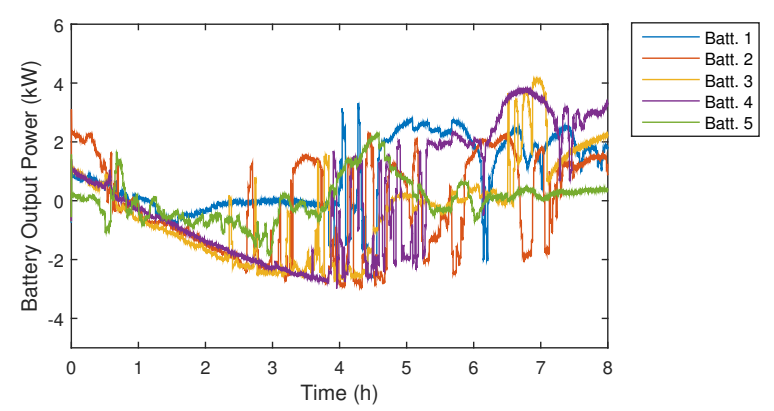

(b) Multi-Agent DOPF.

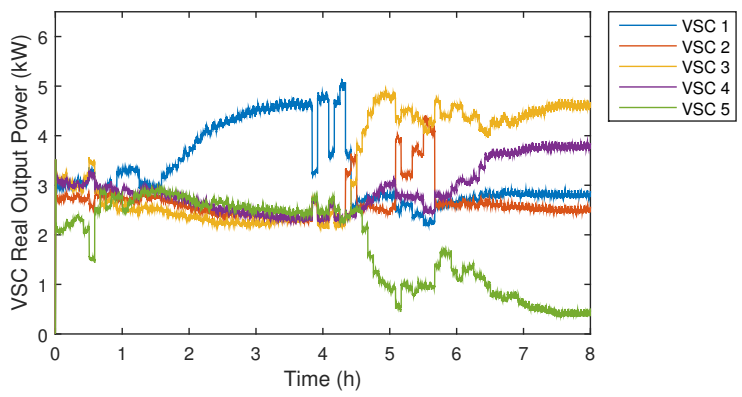

(b) Multi-Agent DOPF.

QP projection problems for the multi-agent DOPF strategy (with the microgrid agents implemented virtually on a single computer). The average solution time for the centralised optimisation problem is $0.0282 \mathrm{~s}$ on an Intel Core i7-4770 CPU. The average solution time for the agent projection problems is $0.0078 \mathrm{~s}$. The distributed primal subgradient algorithm was implemented with 500 communication sub-intervals between each MPC sampling instant. This requires $500 \times 0.0078=3.9$ seconds ( $1.3 \%$ of the 5 minute MPC sampling period), leaving time for communication delays and overheads. The step-size was chosen as $\alpha(\kappa)=\frac{0.01}{1+\kappa}$, which satisfies (28). TCP/IP communication over an Ethernet network was used between the computer running the MPC strategy and the real-time digital simulator, which simulates the microgrid and the lower level converter controllers. 


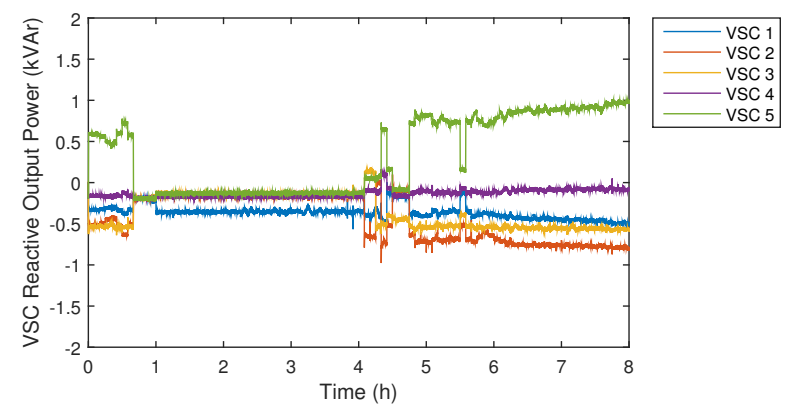

(a) Centralised DOPF.

Fig. 9. VSC reactive output powers.

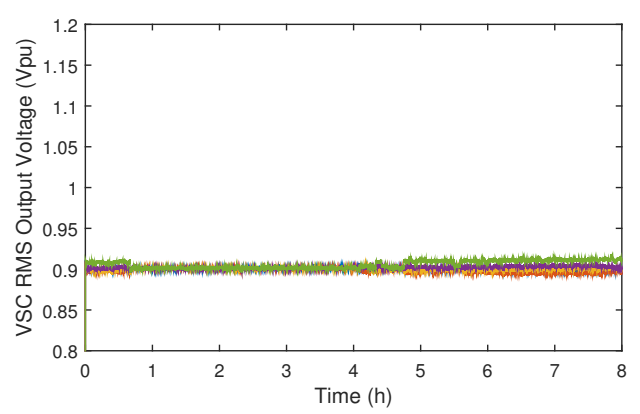

(a) Centralised DOPF.

Fig. 10. VSC RMS output voltages.

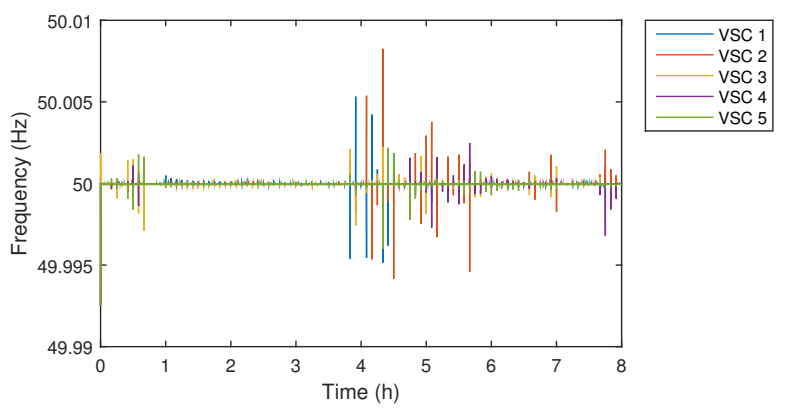

(a) Centralised DOPF.

Fig. 11. VSC output frequencies.

Fig. 5 shows the intermittent generation of the five PV sources operating under maximum power point tracking.

Under the centralised and multi-agent DOPF strategies, the VSC output voltage references are updated every 5 minutes, with the goal of minimising power consumption within power quality and device operating limits. The batteries charge/discharge as required to balance the difference between the PV generation and the real power exported to the microgrid. The batteries begin with SoC levels between the limits of 40\% and 100\%, as shown in Fig. 6(a) and Fig. 6(b). The battery output powers for the centralised and multi-agent DOPF strategies are shown in Fig. 7(a) and Fig. 7(b). Fig. 8(a) and Fig. 8(b) show the real power exported to the microgrid. Fig. 9(a) and Fig. 9(b) show the reactive power exported to the microgrid.

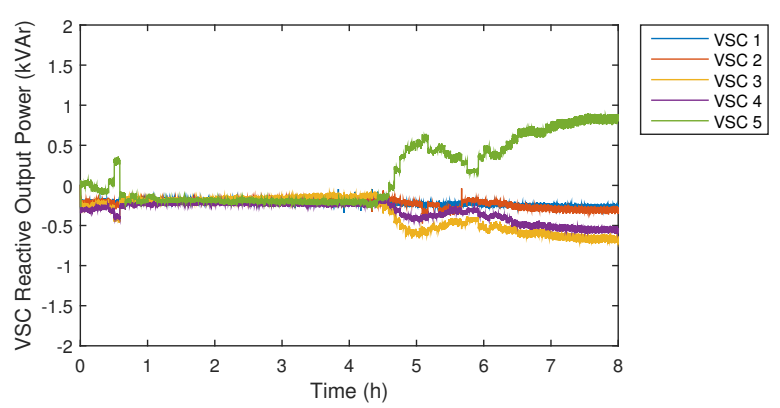

(b) Multi-Agent DOPF.

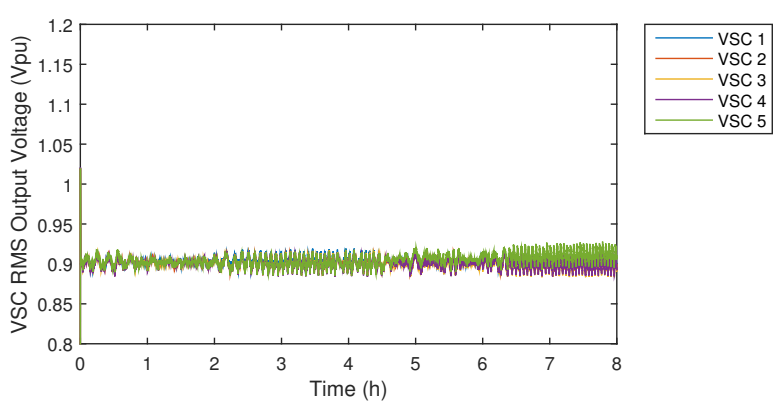

(b) Multi-Agent DOPF.

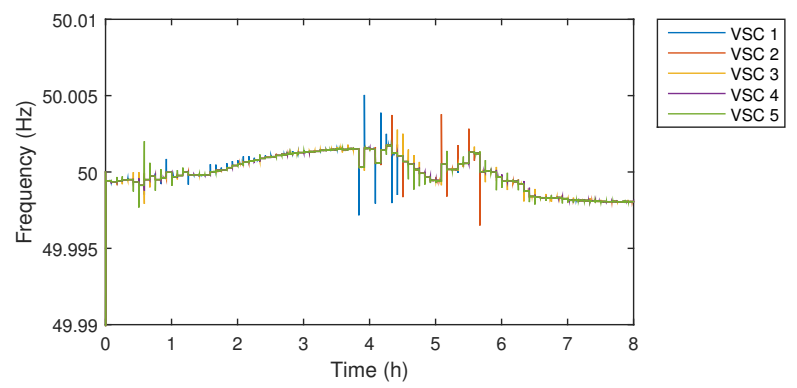

(b) Multi-Agent DOPF.

Fig. 6(a) shows that under the centralised DOPF strategy, the minimum SoC level reached by any of the batteries is $38.9 \%$, and the maximum $\mathrm{SoC}$ is $102.2 \%$. These slight violations of SoC limits of $40 \%$ to $100 \%$ are caused by the linear power flow approximations, and the 5 minute sampling period. Under the multi-agent DOPF strategy, the limited number of communication sub-intervals means the central solution is not fully reached, causing larger SoC constraint violations. As shown in Fig. 6(b), the SoC levels of the batteries vary between $37.9 \%$, and $102.3 \%$.

Battery utilisation can also be compared using energy throughput i.e. the total energy discharged from the batteries. The combined energy throughput of the batteries is $50.85 \mathrm{kWh}$ under the centralised DOPF strategy and $50.67 \mathrm{kWh}$ under the multi-agent DOPF strategy. 
Under both the centralised and multi-agent DOPF strategies, the VSC output voltages remain near the lower limit of $0.9 \mathrm{pu}$, which reduces power consumption since the microgrid load is primarily resistive. The VSC output voltages are shown in Fig. 10(a) and Fig. 10(b). Fig. 11(a) and Fig. 11(b) show the VSC frequencies under the primary droop control strategy.

The multi-agent DOPF strategy provides VSC references that are further from an exact power balance, causing a reduction in power quality. This can be quantified in terms of the mean deviations of the VSC output voltages and frequencies from their nominal values. The mean VSC output voltage deviation is $1.66 \mathrm{~V}(0.0040 \mathrm{pu})$ under the centralised DOPF strategy and $2.64 \mathrm{~V}(0.0064 \mathrm{pu})$ under the multi-agent DOPF strategy. The mean frequency deviation is $4.8 \times 10^{-5} \mathrm{~Hz}$ under the centralised DOPF strategy and $9.4 \times 10^{-4} \mathrm{~Hz}$ under the multi-agent DOPF strategy.

The multi-agent DOPF strategy also provides slightly suboptimal performance in terms of the power consumption minimisation objective, but is able to approach the performance of the centralised optimisation. The average microgrid power consumption is $15.49 \mathrm{~kW}$ under the centralised DOPF strategy, and $15.51 \mathrm{~kW}(0.13 \%$ higher $)$ under the multi-agent DOPF strategy.

\section{Vi. Conclusion}

A multi-agent DOPF strategy has been presented for microgrids with distributed ES systems. This removes the need for a central energy management system, providing a scalable solution for future power networks, which are expected to include many small distributed renewable sources and ES systems. The information required by each agent is independent of the communication network topology, providing increased flexibility and robustness. The performance of the proposed control strategy was verified using an RTDS real-time digital simulator, showing the proper interaction between the multiagent MPC implementation and the low level converter controllers.

\section{APPENDIX}

Constant power loads can also be included in the static synchronous d-q reference frame voltage-current model. Consider a load at bus $i$ with real and reactive power components,

$$
\begin{aligned}
P_{\text {loadi }} & =v_{\text {bdi }} i_{\text {loaddi }}+v_{\text {bqi }} i_{\text {loadqi }} \\
Q_{\text {loadi }} & =v_{\text {bdi }} i_{\text {loadqi }}-v_{\text {bqi }} i_{\text {loaddi }}
\end{aligned}
$$

In this case, the load current d-q components are given by,

$$
\begin{aligned}
i_{\text {loaddi }}= & \frac{v_{\text {bdi }} P_{\text {loadi }}-v_{\text {bqi }} Q_{\text {loadi }}}{v_{b d i}^{2}+v_{b q i}^{2}}, \\
i_{\text {loadqi }} & =\frac{v_{\text {bqi }} P_{\text {loadi }}+v_{\text {bdi }} Q_{\text {loadi }}}{v_{b d i}^{2}+v_{b q i}^{2}} .
\end{aligned}
$$

Power flow linearisations are introduced to maintain a convex formulation of the DOPF problem. Let the d-q components of the nominal operating voltage at bus $i$ be $\left(V_{b d i}, V_{b q i}\right)$.
The following affine approximations for the load current dq components are obtained.

$$
\begin{aligned}
i_{\text {loaddi }} \approx & {\left[\frac{P_{\text {loadi }}\left(V_{b q i}^{2}-V_{b d i}^{2}\right)+2 Q_{\text {loadi }} V_{b d i} V_{b q i}}{\left(V_{b d i}^{2}+V_{b q i}^{2}\right)^{2}}\right] v_{b d i}, } \\
& +\left[\frac{\left.Q_{\text {loadi }}\left(V_{b q i}^{2}-V_{b d i}^{2}\right)-2 P_{\text {loadi }} V_{b d i} V_{b q i}\right]}{\left(V_{b d i}^{2}+V_{b q i}^{2}\right)^{2}}\right] v_{b q i} \\
& +\frac{2\left(P_{\text {loadi }} V_{b d i}-Q_{\text {loadi }} V_{b q i}\right)}{\left(V_{b d i}^{2}+V_{b q i}^{2}\right)^{2}} . \\
i_{\text {loadqi }} \approx & {\left[\frac{Q_{\text {loadi }}\left(V_{b q i}^{2}-V_{b d i}^{2}\right)-2 P_{\text {loadi }} V_{b d i} V_{b q i}}{\left(V_{b d i}^{2}+V_{b q i}^{2}\right)^{2}}\right] v_{b d i} } \\
& +\left[\frac{P_{\text {loadi }}\left(V_{b d i}^{2}-V_{b q i}^{2}\right)-2 Q_{\text {loadi }} V_{b d i} V_{b q i}}{\left(V_{b d i}^{2}+V_{b q i}^{2}\right)^{2}}\right] v_{b q i} \\
& +\frac{2\left(P_{\text {loadi }} V_{b q i}+Q_{\text {loadi }} V_{b d i}\right)}{\left(V_{b d i}^{2}+V_{b q i}^{2}\right)^{2}}
\end{aligned}
$$

The linear functions for the microgrid voltages and currents from (4) become affine functions of the microgrid bus voltages.

\section{REFERENCES}

[1] M. S. Whittingham, "History, Evolution, and Future Status of Energy Storage," Proceedings of the IEEE, vol. 100, no. Special Centennial Issue, pp. 1518-1534, May 2012.

[2] R. H. Lasseter, "MicroGrids," in 2002 IEEE Power Engineering Society Winter Meeting. Conference Proceedings (Cat. No.02CH37309), vol. 1, 2002, pp. 305-308.

[3] D. E. Olivares, A. Mehrizi-Sani, A. H. Etemadi, C. A. Canizares, R. Iravani, M. Kazerani, A. H. Hajimiragha, O. Gomis-Bellmunt, M. Saeedifard, R. Palma-Behnke, G. A. Jimenez-Estevez, and N. D. Hatziargyriou, "Trends in Microgrid Control," IEEE Trans. Smart Grid, vol. 5, no. 4, pp. 1905-1919, July 2014.

[4] J. A. Momoh, R. Adapa, and M. E. El-Hawary, "A review of selected optimal power flow literature to 1993. I. Nonlinear and quadratic programming approaches," IEEE Trans. Power Syst., vol. 14, no. 1, pp. 96-104, Feb. 1999.

[5] Y. Levron, J. M. Guerrero, and Y. Beck, "Optimal Power Flow in Microgrids With Energy Storage," IEEE Trans. Power Syst., vol. 28, no. 3, pp. 3226-3234, Aug. 2013.

[6] T. A. Nguyen and M. L. Crow, "Stochastic Optimization of RenewableBased Microgrid Operation Incorporating Battery Operating Cost," IEEE Trans. Power Syst., vol. PP, no. 99, pp. 1-8, 2015.

[7] D. E. Olivares, C. A. Canizares, and M. Kazerani, "A Centralized Energy Management System for Isolated Microgrids," IEEE Trans. Smart Grid, vol. 5, no. 4, pp. 1864-1875, July 2014.

[8] A. Parisio, E. Rikos, and L. Glielmo, "A Model Predictive Control Approach to Microgrid Operation Optimization," IEEE Trans. Control Syst. Technol., vol. 22, no. 5, pp. 1813-1827, Sept. 2014.

[9] Y. Wang, K. T. Tan, and P. L. So, "Coordinated control of battery energy storage system in a microgrid," in 2013 IEEE PES Asia-Pacific Power and Energy Engineering Conference (APPEEC), Dec. 2013, pp. 1-6.

[10] E. Perez, H. Beltran, N. Aparicio, and P. Rodriguez, "Predictive Power Control for PV Plants With Energy Storage," IEEE Trans. Sustain. Energy, vol. 4, no. 2, pp. 482-490, Apr. 2013.

[11] E. Mayhorn, K. Kalsi, M. Elizondo, N. Samaan, and K. Butler-Purry, "Optimal control of distributed energy resources using model predictive control," in 2012 IEEE Power and Energy Society General Meeting, July 2012, pp. 1-8.

[12] M. Khalid and A. V. Savkin, "A model predictive control approach to the problem of wind power smoothing with controlled battery storage," Renewable Energy, vol. 35, no. 7, pp. 1520-1526, July 2010.

[13] H. Dagdougui, L. Dessaint, and A. Ouammi, "Optimal power exchanges in an interconnected power microgrids based on model predictive control," 2014 IEEE PES General Meeting - Conference \& Exposition, pp. 1-5, July 2014. 
[14] F. Garcia-Torres and C. Bordons, "Optimal Economical Schedule of Hydrogen-Based Microgrids With Hybrid Storage Using Model Predictive Control," IEEE Trans. Ind. Electron., vol. 62, no. 8, pp. 5195-5207, Aug. 2015.

[15] P. P. Zeng, Z. Wu, X.-P. Zhang, C. Liang, and Y. Zhang, "Model predictive control for energy storage systems in a network with high penetration of renewable energy and limited export capacity," 2014 Power Systems Computation Conference, pp. 1-7, Aug. 2014.

[16] D. J. Hill, T. Liu, and G. Verbic, "Smart grids as distributed learning control," in 2012 IEEE Power and Energy Society General Meeting, July 2012, pp. 1-8.

[17] Y. Simmhan, A. G. Kumbhare, B. Cao, and V. Prasanna, "An Analysis of Security and Privacy Issues in Smart Grid Software Architectures on Clouds," in 2011 IEEE 4th International Conference on Cloud Computing, July 2011, pp. 582-589.

[18] F. L. Lewis, H. Zhang, K. Hengster-Movric, and A. Das, Cooperative Control of Multi-Agent Systems, ser. Communications and Control Engineering. London: Springer London, 2014.

[19] S. D. J. McArthur, E. M. Davidson, V. M. Catterson, A. L. Dimeas, N. D. Hatziargyriou, F. Ponci, and T. Funabashi, "Multi-Agent Systems for Power Engineering Applications-Part I: Concepts, Approaches, and Technical Challenges," IEEE Trans. Power Syst., vol. 22, no. 4, pp. 1743-1752, Nov. 2007.

[20] A. Bidram, A. Davoudi, F. L. Lewis, and J. M. Guerrero, "Distributed Cooperative Secondary Control of Microgrids Using Feedback Linearization," IEEE Trans. Power Syst., vol. 28, no. 3, pp. 3462-3470, Aug. 2013.

[21] J. W. Simpson-Porco, F. Dörfler, and F. Bullo, "Synchronization and power sharing for droop-controlled inverters in islanded microgrids," Automatica, vol. 49, no. 9, pp. 2603-2611, Sept. 2013.

[22] V. Nasirian, S. Moayedi, A. Davoudi, and F. L. Lewis, "Distributed Cooperative Control of DC Microgrids," IEEE Trans. Power Electron., vol. 30, no. 4, pp. 2288-2303, Apr. 2015.

[23] T. Morstyn, B. Hredzak, and V. G. Agelidis, "Distributed Cooperative Control of Microgrid Storage," IEEE Trans. Power Syst., vol. 30, no. 5, pp. 2780-2789, Sept. 2015.

[24] T. Morstyn, B. Hredzak, and V. G. Agelidis, "Cooperative MultiAgent Control of Heterogeneous Storage Devices Distributed in a DC Microgrid," IEEE Trans. Power Syst., vol. 31, no. 4, pp. 2974-2986, Sept. 2015.

[25] T. Morstyn, B. Hredzak, G. D. Demetriades, and V. G. Agelidis, "Unified Distributed Control for DC Microgrid Operating Modes," IEEE Trans. Power Syst., vol. 31, no. 1, pp. 802-812, Jan. 2016.

[26] C. Li, T. Dragičević, M. G. Plaza, F. Andrade, J. C. Vásquez, and J. M. Guerrero, "Multiagent based distributed control for state-of-charge balance of distributed energy storage in DC microgrids," in IECON 2014 - 40th Annual Conference of the IEEE Industrial Electronics Society, Oct. 2014, pp. 2180-2184.

[27] C. Li, T. Dragičević, J. C. Vásquez, J. M. Guerrero, and E. A. A. Coelho, "Multi-agent-based distributed state of charge balancing control for distributed energy storage units in AC microgrids," in 2015 IEEE Applied Power Electronics Conference and Exposition (APEC), Mar. 2015, pp. 2967-2973.

[28] W. Shi, X. Xie, C.-c. Chu, and R. Gadh, "A distributed optimal energy management strategy for microgrids," in 2014 IEEE International Conference on Smart Grid Communications (SmartGridComm), vol. 6, no. 3, Nov. 2014, pp. 200-205.

[29] A. Cortés and S. Martínez, "On distributed reactive power and storage control on microgrids," International Journal of Robust and Nonlinear Control, Jan. 2016

[30] M. Kraning, E. Chu, J. Lavaei, and S. Boyd, "Dynamic Network Energy Management via Proximal Message Passing," Foundations and Trends in Optimization, vol. 1, no. 2, pp. 70-122, Nov. 2014.

[31] G. Hug, S. Kar, and C. Wu, "Consensus+Innovations Approach for Distributed Multiagent Coordination in a Microgrid," IEEE Trans. Smart Grid, vol. 6, no. 4, pp. 1893-1903, July 2015.

[32] S. Papathanassiou, N. Hatziargyriou, and K. Strunz, "A Benchmark Low Voltage Microgrid Network," Proceedings of the CIGRE Symposium: Power Systems with Dispersed Generation, pp. 1-8, Apr. 2005.

[33] N. Pogaku, M. Prodanovic, and T. C. Green, "Modeling, Analysis and Testing of Autonomous Operation of an Inverter-Based Microgrid," IEEE Trans. Power Electron., vol. 22, no. 2, pp. 613-625, Mar. 2007.

[34] Zhu, M. and S. Martinez, "On Distributed Convex Optimization Under Inequality and Equality Constraints," IEEE Trans. Autom. Control, vol. 57, no. 1, pp. 151-164, Jan. 2012.

[35] M. Diaz-Aguiló, J. Sandraz, R. Macwan, F. de León, D. Czarkowski, C. Comack, and D. Wang, "Field-validated load model for the analysis of CVR in distribution secondary networks: Energy conservation," IEEE Trans. Power Del., vol. 28, no. 4, pp. 2428-2436, Oct. 2013.

[36] Z. Wang, M. Begovic, and J. Wang, "Analysis of conservation voltage reduction effects based on multistage SVR and stochastic process," IEEE Trans. Smart Grid, vol. 5, no. 1, pp. 431-439, Jan. 2014.

[37] Z. Wang and J. Wang, "Review on implementation and assessment of conservation voltage reduction," IEEE Trans. Power Syst., vol. 29, no. 3, pp. 1306-1315, May 2014.

[38] Z. Wang and J. Wang, "Time-varying stochastic assessment of conservation voltage reduction based on load modeling," IEEE Trans. Power Syst., vol. 29, no. 5, pp. 2321-2328, Sept. 2014.

[39] Z. Wang, B. Chen, J. Wang, and M. M. Begovic, "Stochastic DG Placement for Conservation Voltage Reduction Based on Multiple Replications Procedure," IEEE Trans. Power Del., vol. 30, no. 3, pp. 10391047, June 2015.

[40] B. Zhao, X. Zhang, J. Chen, C. Wang, and L. Guo, "Operation optimization of standalone microgrids considering lifetime characteristics of battery energy storage system," IEEE Trans. Sustain. Energy, vol. 4, no. 4, pp. 934-943, 2013.

[41] R. Dufo-López, J. M. Lujano-Rojas, and J. L. Bernal-Agustín, "Comparison of different lead-acid battery lifetime prediction models for use in simulation of stand-alone photovoltaic systems," Applied Energy, vol. 115, pp. 242-253, Feb. 2014.

[42] T. Kim and W. Qiao, "A Hybrid Battery Model Capable of Capturing Dynamic Circuit Characteristics and Nonlinear Capacity Effects," IEEE Trans. Energy Convers., vol. 26, no. 4, pp. 1172-1180, Dec. 2011.

[43] M. Diagne, M. David, P. Lauret, J. Boland, and N. Schmutz, "Review of solar irradiance forecasting methods and a proposition for small-scale insular grids," Renewable and Sustainable Energy Reviews, vol. 27, pp. 65-76, Nov. 2013.

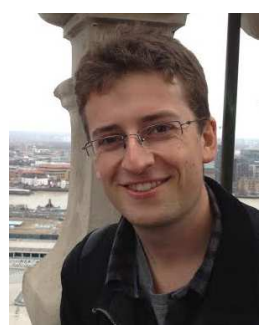

Thomas Morstyn (S'13) received the B.E. (Hon.) degree in electrical engineering from the University of Melbourne, Australia, in 2011.

$\mathrm{He}$ worked as an electrical engineer in the Rio Tinto Technology and Innovation group for two years. He is currently working towards the Ph.D. degree at the Australian Energy Research Institute, The University of New South Wales, Sydney, NSW, Australia. His current research interests include multiagent control and optimisation for the integration of distributed renewable generation and energy storage systems into power networks.

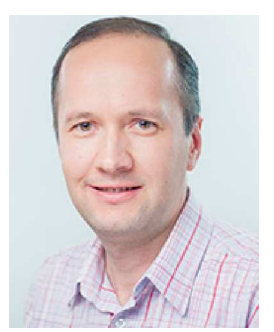

Branislav Hredzak (M'98-SM'13) received the B.Sc./M.Sc. degree from the Technical University of Kosice, Slovak Republic, in 1993, and the Ph.D. degree from Napier University of Edinburgh, U.K., in 1997, all in electrical engineering.

He was a Lecturer and a Senior Researcher in Singapore from 1997 to 2007 . He is currently a Senior Lecturer in the School of Electrical Engineering and Telecommunications, The University of New South Wales, Sydney, NSW, Australia. His current research interests include hybrid storage technologies and advanced control systems for power electronics and storage systems.

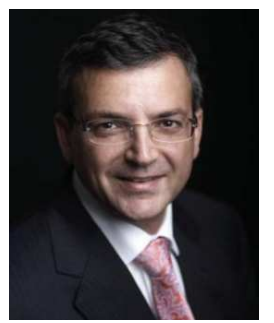

Vassilios G. Agelidis (S'89-M'91-SM'00-F'16) was born in Serres, Greece. He received the B.Eng. degree in electrical engineering from the Democritus University of Thrace, Thrace, Greece, in 1988, the M.S. degree in applied science from Concordia University, Montreal, QC, Canada, in 1992, and the $\mathrm{Ph} . \mathrm{D}$. degree in electrical engineering from Curtin University, Perth, Australia, in 1997. He has worked at Curtin University (1993-1999), University of Glasgow, U.K. (2000-2004), Murdoch University, Perth, Australia (2005-2006), the University of Sydney, Australia (2007-2010), and the University of New South Wales (UNSW), Sydney, Australia (2010-2016). He is currently a professor at the Department of Electrical Engineering, Technical University of Denmark.

Dr. Agelidis received the Advanced Research Fellowship from the U.K.'s Engineering and Physical Sciences Research Council in 2004. He was the Vice-President Operations within the IEEE Power Electronics Society from 2006 to 2007. He was an AdCom Member of the IEEE Power Electronics Society from 2007 to 2009 and the Technical Chair of the 39th IEEE Power Electronics Specialists Conference, Rhodes, Greece, 2008. 